\title{
Application of Rasch model in validating the content of measurement instrument for blog quality
}

\begin{abstract}
Research in blog quality is very crucial nowadays in order to have a good quality blog in the blogosphere. The blog quality criteria have been derived from a rigorous metadata analysis. Yet, these criteria have not been reviewed and their significance has not been proven systematically. In this paper, Rasch Model is applied to produce an empirical evidence of content validity of the blog quality criteria. This study confirms that the definitions of 11 families and the 49 criteria assigned have content validity by mean of online survey. These criteria will then be used as a basis of constructing the instrument to measure the acceptability of the criteria for blog quality.
\end{abstract}

Keyword: Blog quality; Content validity; Rasch model 